\title{
Toma de apuntes y aprendizaje en estudiantes de Educación Superior
}

\section{Note-taking and learning in higher education students}

\author{
Dagoberto SALGADO-HORTA y Alexander MAZ-MACHADO \\ Universidad del Tolima- Universidad de Córdoba
}

Recibido: Septiembre 2012

Aceptado: Noviembre 2013

\section{Resumen}

Se realizó una investigación con estudiantes de la asignatura de Estadística de diferentes instituciones universitarias, en relación con la utilización de estrategias de aprendizaje para registrar la información. Tomando como marco de referencia las teorías constructivistas del aprendizaje, el objetivo del trabajo se centra en comprobar el impacto de determinadas estrategias de aprendizaje en el proceso de toma de apuntes (note-taking) por parte de los estudiantes durante el aprendizaje.

Se trabajó con tres temas de una unidad didáctica de la asignatura de Estadística, tomando tres mediciones: la primera sin la utilización de estrategias de aprendizaje en la toma de apuntes, la segunda después de utilizar la estrategia $\mathrm{A}$, y la tercera después de utilizar la estrategia $\mathrm{B}$. Las mediciones se realizaron a dos grupos experimentales y un grupo control, mediante preguntas de selección múltiple con única respuesta y medidas con una calificación de 0 a 5 . Los resultados obtenidos muestran que en los grupos donde los estudiantes utilizan las estrategias de aprendizaje seleccionadas para tomar apuntes mejoran su nivel de respuesta, lo cual no se produce en el grupo control.

Palabras clave: Estrategias de aprendizaje, toma de apuntes, aprendizaje significativo, enseñanza superior.

\begin{abstract}
Research was conducted with students from a statistics course in different institutions of higher education in relation to the use of learning strategies to record information. Taking as reference the constructivist learning theories, the objective of the work is focused on testing the impact of specific learning strategies in the process of note-taking by students during learning.

We worked with three themes of a teaching unit of a statistics course, taking three measurements: the first without the use of learning strategies in note-taking, the second after using the strategy A, and the third after using the strategy B. The measurements were performed at two experimental groups and one control group, using multiple choice questions with single response and measures with a score of 0-5. The results show that in groups where students use learning strategies selected for notetaking improved response rate, which does not occur in the control group.
\end{abstract}

Keywords: learning strategies, note-taking, meaningful learning, higher education. 
Las instituciones de educación superior en Colombia se clasifican en Instituciones Técnicas Profesionales, Instituciones Tecnológicas, Instituciones Universitarias o Escuelas Tecnológicas y las Universidades. Todas ellas han tratado de ajustarse a los requerimientos que les plantea su entorno socioeconómico y cultural. Esto les ha permitido desarrollarse y mantener su vigencia como instituciones sociales a lo largo de su historia. La época actual, caracterizada por fenómenos de globalización, rápido desarrollo científico y tecnológico, así como la diversificación de las fuentes de acceso al conocimiento, entre otros, ha generado la necesidad de desarrollar importantes modificaciones en sus funciones, particularmente en lo que concierne a su tarea de generación y distribución social de conocimiento.

Los nuevos enfoques en cuanto a la construcción de conocimiento se orientan básicamente a enfatizar la importancia de la contextualización del saber producido, por una parte, y por otra, a la generación de nuevas estrategias en los actores que producen y los que se apropian de éste para utilizarlo en situaciones concretas. Estos cambios no se limitan a las formas en que se produce conocimiento, sino que alcanza, de manera ineludible, a las modalidades a través de las cuales se organiza pedagógicamente y se hace llegar a los individuos, a través de prácticas y dinámicas de enseñanza y de aprendizaje (Rosário, Mourão, Núñez, González-Pienda, Solano y Valle, 2007; Pérez, Valenzuela, Díaz, González-Pienda y Núñez, 2011). El contexto actual demanda formar personas preparadas para enfrentar nuevas necesidades, por lo que las actividades académicas tradicionales, particularmente la enseñanza, se han visto rebasadas en cuanto a su posibilidad de cumplir con esta tarea.

Se reconoce internacionalmente que los modelos vigentes de formación profesional resultan inadecuados ante las características del nuevo contexto (UNESCO, 2001). En parte es debido a que la formación se ha limitado al espacio escolar, en donde los estudiantes consumen cierta información adquiriendo habilidades, actitudes y valores implicados en los perfiles establecidos. Todo ello, con base en ciertas suposiciones acerca del individuo, de sus procesos cognitivos y socio-afectivos, así como de la actividad que realizará como futuro profesional. Este modelo universitario centrado en la enseñanza empieza a cambiar hacia un modelo centrado en el aprendizaje y tal cambio implica un cambio cultural dentro del ámbito universitario como ya viene sucediendo en otros países (Fernández, 2006).

Por tanto, las instituciones educativas tienen la responsabilidad de generar condiciones que posibiliten a los individuos lograr aprendizajes relevantes para lograr su inserción social y productiva así como la construcción de una identidad personal, dentro de un contexto sociocultural determinado. Para ello, están obligadas a incorporar alternativas innovadoras que impulsen la apropiación del conocimiento, transformando su organización interna, articulándose de manera más pertinente con la comunidad y con el mercado, creando redes académicas con otras instituciones. Una de estas alternativas es fomentar el aprendizaje significativo, a través de diversas estrategias durante el proceso de aprendizaje. 


\section{Marco teórico}

El aprendizaje se ha convertido en uno de los mayores desafíos de la sociedad. Si el individuo se mueve en un mundo cada vez más complejo y cambiante, no puede conformarse con adquirir las destrezas básicas ni un conjunto predeterminado y finito de saberes adquiridos en un escenario escolarizado. Tiene que aprender a lo largo de toda su vida en diversos espacios y debe aprender a sacar provecho de su experiencia, de sus éxitos y fracasos así como transferirlos a nuevos contextos para resolver los retos que se le plantean (Esteve, 2003; Enríquez y Rentería, 2007). De tal forma, que como señalan Muñoz y Gómez (2005, p. 418), para los docentes universitarios un tema básico de su trabajo debería ser "[...] el análisis de cuáles son los procesos y estrategias a través de los cuales los estudiantes aprenden"

El proceso de aprendizaje en las instituciones educativas puede ser analizado a partir de los procesos psicológicos que el sujeto pone en juego para aprender con base en un conjunto de mecanismos sociales y culturales susceptibles de generar condiciones que apoyan el aprendizaje y ofrecen la posibilidad de explicar e intervenir en el fenómeno educativo, particularmente en los aprendizajes, desde diversas disciplinas: psicología, sociología, antropología y en general, las incluidas en el campo de las ciencias de la educación. Las distintas concepciones del aprendizaje que fundamentan el currículo en las instituciones educativas (conductismo, constructivismo, psicología sociocultural, algunas teorías instruccionales, entre otras) así como las estrategias de intervención para mejorarlo, expresan la convergencia de distintas perspectivas y enfoques psicológicos. Son abundantes los estudios que señalan el interés y la importancia de la investigación sobre la educación superior (Brown y Glasner, 2003; Ibarra y Rodríguez, 2010) y esto convierte la evaluación del aprendizaje en algo estratégico para la universidad de hoy (Gibbs, 2003).

Por lo tanto en la universidad se debe procurar por enseñar técnicas y estrategias que permitan al estudiante alcanzar y comprender los conocimientos necesarios para su desempeño social como profesional. Una de las múltiples opciones por la que se puede apostar es la toma de notas (note-taking). Enseñar al estudiante universitario estrategias para anotar los contenidos disciplinares dados durante las sesiones de clase no significa, desde nuestra óptica, únicamente «entrenarlo» en la utilización de procedimientos de registro de datos. Por el contrario, significa ayudarle a adquirir conciencia del objetivo de la toma de apuntes y de las condiciones específicas en que debe efectuarse, con el fin de que decida el tipo de anotación que empleará y regule en todo momento su aplicación.

Hoy por hoy a nadie escapa que los procedimientos de anotación o registro de la información escrita constituyen el método de transcripción hegemónico en la enseñanza superior. Los psicólogos educativos señalan que la toma de notas en una asignatura cumple dos funciones: una para la revisión y la otra como ayuda a los estudiantes para aprender el tema abordado (Kiewra, 1989; Van Meter, Yokoi y Pressley, 1994).

En una encuesta realizada por Hartley y Davies (1978), a estudiantes norteamericanos y británicos, respecto al por qué tomaban notas durante las clases, 
éstos expusieron principalmente dos razones: la primera, relacionada con el proceso de recoger la información, porque los estudiantes consideraban que escribir las ideas expresadas por el profesor con palabras propias, permitía organizar e interpretar el material que se estaba presentando; la segunda, relacionada con la utilización posterior de las notas recogidas, dado que los estudiantes opinaron que podía ser útil consultar los apuntes posteriormente para estudiar antes de un examen, para obtener una puntuación más alta y un mejor rendimiento académico. La toma de notas es una actividad estudiantil importante que en si misma puede ser una técnica en la parte del proceso de aprendizaje (War y Tatsukawa, 2003; Kobayashi, 2005).

Las investigaciones sobre el tema han clasificado hasta seis clases estrategias en la toma de notas: Absorción, mantenimiento, traslación descodificación, integración y organización (Teng, 2011). Estas se pueden dar individualmente o de manera conjunta alguna de ellas. Los estudiantes consideran que el registro de la información puede ser el resultado de un análisis e interpretación personal y posibilita la reconstrucción y recuerdo posterior del material recogido. Ellos toman decisiones acerca de qué elementos de una conferencia valen la pena ser escritos, influenciados tanto por la interpretación personal de la información recibida como por las técnicas de toma de notas que poseen (Badger, White, Sutherland y Haggis, 2001).

Si bien es cierto que una gran mayoría de estudiantes toman notas, también lo es que en un porcentaje elevado de casos, no se ha enseñado explícitamente a los estudiantes cómo hacerlo para obtener el máximo beneficio de esta actividad (Badger et al., 2001). Sin embargo, cuando los estudiantes son expuestos de manera sistemática a un entrenamiento en técnicas de toma de notas hay un progreso en los resultados (Kirkgöz, 2010; Slotte y Lonka, 1999). Es éste, uno de los motivos que han llevado al interés por el tema y a diseñar una investigación que permita profundizar en algunos aspectos de la toma de apuntes. Por lo tanto, esta investigación pretende ser una aportación en este sentido.

\section{Objetivo}

Esta investigación tiene como propósito comprobar si el uso de algunas técnicas de toma de apuntes favorece el aprendizaje significativo en temas de Estadística en los estudiantes de educación superior.

\section{Metodología}

\section{Población y muestra}

La población de este estudio la constituyeron estudiantes de ambos sexos de tres instituciones de educación superior de carácter privado, y público ubicadas en la ciudad de Ibagué, Colombia. La muestra estuvo conformada por 80 estudiantes cuya edad osciló entre 18 y 23 años. Todos ellos pertenecían a tres grupos del cuarto semestre (segundo año) donde se impartió la asignatura de Estadística de las tres instituciones: Universidad Cooperativa de Colombia, Universidad del Tolima y la 
Escuela Superior de Administración Pública. No hubo asignación al azar de los sujetos. Ellos pertenecían a grupos que se estaban formando en las instituciones educativas en la asignatura Estadística, por lo tanto la muestra es intencional y por conveniencia.

\section{Diseño}

La muestra se distribuyó en tres grupos naturales que correspondían, en cada caso, a los grupos-clase. El número de estudiantes en cada grupo fue el siguiente: Grupo 1: N= 22. Grupo 2: $\mathrm{N}=30$. Grupo 3: $\mathrm{N}=28$. El grupo 1 es considerado como el grupo control, el grupo 2 como grupo experimental A (aplicación estrategia A) y el grupo 3 como grupo experimental B (aplicación estrategia B).

Se partió de un diseño cuasi-experimental de grupos de comparación no equivalente. Los grupos se formaron en las tres instituciones educativas seleccionadas en la asignatura Estadística. Se manipuló deliberadamente al menos una variable independiente para ver su efecto y relación con una o más variables dependientes, difieren de los experimentos "verdaderos" en el grado de seguridad o confiabilidad que pueda tenerse sobre la equivalencia inicial de los grupos. En nuestro caso hubo un grupo control y dos grupos experimentales.

Para la homologación de la muestra, todos los grupos fueron seleccionados mediante un riguroso sistema de pareo en cuanto a las variables: edad, sexo. Se aclara que los temas tratados fueron idénticos, e impartidos por el mismo docente en cada una de las instituciones.

Este tipo de diseños ha sido muy utilizado en la investigación social y son fácilmente interpretables (Cook y Campbell, 1979). Para la construcción del diseño se utilizan uno o varios grupos a los que se les aplican la variable independiente (la intervención o tratamiento) y de uno o varios grupos de control (que no reciben la intervención o tratamiento). En unos u otros grupos se realizan medidas pre y post tratamiento. El ejemplo prototípico sería el siguiente:

\begin{tabular}{|l|c|c|c|c|}
\hline \multirow{2}{*}{ Grupos } & \multirow{2}{*}{ Asignación } & \multicolumn{3}{|c|}{ Secuencia de registro } \\
\cline { 3 - 5 } & & Pretest & Tratamiento & Postest \\
\hline Experimental (GE) & NA & $\mathrm{Y}_{E l}$ & $\mathrm{X}$ & $\mathrm{Y}_{E t}$ \\
\cline { 3 - 5 } Control (GC) & \multirow{2}{*}{ NA } & $\mathrm{Y}_{C l}$ & ---- & $\mathrm{Y}_{E t}$ \\
\hline
\end{tabular}

Tabla I. Diseño de la investigación

En relación a este diseño se hace constar los siguientes factores (Ato, 1995):

1. El número de grupos, si es único o múltiple, en cuyo caso se distinguirá normalmente entre: grupo experimental (GE) y grupo control (GC). 
2. La variable de asignación: si no existe (NE), si es aleatoria (A), si no es aleatoria ni conocida (NA) y si no es aleatoria pero si conocida (NAC).

3. Secuencia de tratamiento seguida, donde $(\mathrm{Y})$ representa las observaciones o medidas tomadas antes (pre) o después (post) del tratamiento. El subíndice de $(\mathrm{Y})$ indica el orden de registro y el grupo al que pertenecen. Las $(\mathrm{X})$ representan el tratamiento, (--) ausencia de tratamiento, $(\sim \mathrm{X})$ retirada de tratamiento, $\left(\mathrm{X}_{1}\right.$, $\left.\mathrm{X}_{2}, \mathrm{X}_{3} \ldots \mathrm{X}_{\mathrm{n}}\right)$ gradación de tratamientos, $\left(\mathrm{X}^{+}\right)$tratamiento invertido positivo $\mathrm{y}$ (X-) tratamiento invertido negativo.

Variable Independiente: Estrategias de toma de apuntes.

Variable Dependiente: Aprendizaje Significativo del estudiante.

Variables Intervinientes: Sexo, edad y tipo de institución educativa (Privada o Pública).

Procedimiento: A todos los grupos se les aplicó un pretest en conocimientos de estadística (para determinar homogeneidad en el nivel de conocimientos en los tres grupos) y un postest en conocimientos de estadística (para determinar si existen diferencias significativas entre el grupo control y los dos grupos experimentales, después de la aplicación de las estrategias A y B). Serán escogidos los temas por su riqueza conceptual, por su coherencia lógica y por los nexos con la vida diaria de los estudiantes.

El diseño de la investigación se concretó de la siguiente manera:

GE1 (Estrategia A) Hoja Modelo.

GE2 (Estrategia B) Cuadro Sinóptico.

GC Grupo Control: Procedimientos de anotación y toma de apuntes usualmente empleados por el estudiante que, en términos generales, se centran en el registro literal de la información expuesta.

Para la recogida de la información sobre la adquisición de conocimientos, se realizaron dos cuestionarios de selección múltiple de diez ítems con única respuesta que fueron medidos mediante una calificación de 0 a 5 . Los cuestionarios agrupaban los ítems en tres dimensiones:

1. Propósito general del análisis estadístico y formas en que puede ayudar a encontrar soluciones a problemas en la vida profesional.

2. Procedimientos de tabulación y graficación de las variables de tipo cualitativo y cuantitativo.

3. Conceptos estadísticos de posición, dispersión, asimetría, apuntamiento y forma para resumir la información estadística. 
A los resultados se les aplicó un análisis de varianza para un diseño completamente al azar (ANOVA), para contrastar la existencia de diferencias significativas entre los promedios de las mediciones. La información obtenida fue procesada en un banco de datos creado en el programa SPSS. El tratamiento estadístico requirió estudios de validez y confiabilidad del instrumento, así como determinación de los grupos que correspondían a cada estrategia de aprendizaje. Para encontrar la confiabilidad, los datos se sometieron al coeficiente alfa de Cronbach, en tanto que para la validez de constructo se empleó el análisis factorial en su modalidad de análisis de componentes principales y rotación varimax.

\section{Instrumentos y fases:}

Primera fase. Se considero necesario valorar tres aspectos antes de realizar la intervención:

a) Valoración de los conocimientos previos de los estudiantes en el área de Estadística, para comprobar la homogeneidad entre los grupos en los que se utilizaba una estrategia de enseñanza determinada (grupo-clase) y los grupos de alumnos a los que se les enseñaban diferentes estrategias de aprendizaje.

b) Valoración del aprendizaje de los estudiantes tras recibir una Unidad Didáctica del programa de la asignatura, y antes de cualquier intervención. En todos los grupos la Unidad Didáctica valorada fue la misma y se controlo la duración de ésta y la equivalencia de los contenidos a través de la grabación de todas las clases.

Cabe señalar también, a este respecto, que se realizó una sesión de reflexión en la que se concretaron las ideas sobre el contenido de la Unidad Didáctica y se determinaron los conceptos básicos, la secuenciación de los contenidos y las actividades de enseñanza y evaluación que se llevarían a cabo. Así mismo se negoció y consensuó el significado y el sentido de la aplicación de las estrategias correspondientes a cada grupo-clase.

En ambos apartados, a y b, que se corresponden con los apartados «conocimientos previos» $\mathrm{y}$ «evaluación inicial», se valoro el aprendizaje de los alumnos a través de una escala cuantitativa de 0 a 5 .

c) Análisis de los apuntes de los estudiantes con el fin de comprobar el proceso que seguían para confeccionar los apuntes y si espontáneamente ya utilizaban alguna de las estrategias de aprendizaje que iban a enseñarse. Para ello, se fotocopian los apuntes de los alumnos después de una sesión de clase, sin previo aviso, y se analizan diferenciando las siguientes categorías:

1. Reproducción literal versus interpretación personal.

2. Diferenciación entre ideas principales, secundarias, ejemplos e información complementaria.

3. Anotación de dudas y preguntas; ampliación de la información. 
4. Establecimiento de relaciones significativas con sus conocimientos previos.

5. Resumen de la información más relevante.

Cabe indicar que en ningún caso, durante la fase inicial, los estudiantes utilizaban ninguna de las estrategias que se enseñaron posteriormente para tomar apuntes.

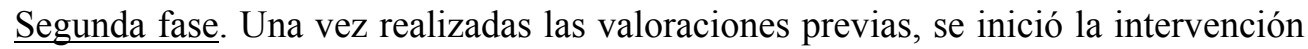
que detallamos a continuación:

Enseñanza de las estrategias de aprendizaje en la toma de apuntes a cada uno de los grupos experimentales. La enseñanza de cada una de las estrategias fue impartida por el mismo profesor en los dos grupos de forma. La dinámica de las sesiones se desarrolló del mismo modo en todos los casos y el contenido de éstas fue el mismo exceptuando el momento en que se explicaba el contenido específico de la estrategia en cuestión. En el grupo control, el contenido de la sesión fue diferente, y consistió en la reproducción de un vídeo irrelevante para el tema, sobre el que podían tomar apuntes, y su posterior comentario. La enseñanza de estas estrategias se realiza en una sesión de dos horas para cada subgrupo y se pide a los estudiantes explícitamente que utilicen la estrategia aprendida para tomar apuntes durante las siguientes clases (correspondientes a la Unidad Didáctica de la intervención).

La dinámica de estas sesiones se desarrollo siguiendo los pasos que detallamos a continuación:

1. En primer lugar los estudiantes tomaron apuntes de un tema de la asignatura de Estadística impartido por el profesor durante un cuarto de hora.

2. A continuación el profesor y los estudiantes valoraron conjuntamente los apuntes resultantes a partir de un conjunto de criterios predeterminado.

3. Seguidamente el profesor informó de la existencia de métodos que podían facilitar la toma de apuntes y el estudio posterior de la materia, e introdujo la información correspondiente a la estrategia de aprendizaje asignada al grupo en cuestión.

4. Después los estudiantes, individualmente, reorganizaron los apuntes tomados inicialmente, en función de las orientaciones dadas por el profesor.

5. A continuación el profesor y los estudiantes, conjuntamente, analizaron los nuevos apuntes, intercambiando opiniones y comentando los aspectos más relevantes. El profesor presentó un modelo de apuntes en función de la estrategia explicada.

6. Seguidamente los estudiantes tomaron de nuevo apuntes sobre un nuevo tema de la asignatura de Estadística, durante un cuarto de hora, utilizando la estrategia de aprendizaje adquirida.

7. Por último los estudiantes y el profesor analizaron las ventajas, dificultades y dudas planteadas por la estrategia de anotación y su correspondiente aplicación. 
Los estudiantes de los grupos experimentales que no utilizaron la estrategia enseñada fueron desestimados como sujetos de la investigación.

Tercera fase: Finalmente, después de la intervención se consideró necesario realizar el siguiente análisis:

a) Valoración del aprendizaje de los estudiantes inmediatamente después de concluir la Unidad Didáctica seleccionada para la intervención. Para ello, se utilizaron test con preguntas tipo múltiple y medidas mediante la escala cuantitativa de 0 a 5 .

b) Comparación del grado de profundidad y significatividad del aprendizaje obtenido después de la Unidad Didáctica inicial y de la Unidad Didáctica tratada experimentalmente.

\section{Resultados}

En relación con los objetivos, el análisis estadístico realizado (ANOVA de un factor del paquete estadístico SPSS y las pruebas de comparaciones múltiples DMS y DUNCAN), han tenido como propósito mostrar las diferencias en el aprendizaje entre los distintos grupos (V.D.) en función de las estrategias de toma involucradas, a través de la comparación de medias. Por lo que respecta al nivel de significancia, hemos utilizado $\alpha=0.01$.

Se analiza en primer lugar las diferencias que muestran los grupos respecto al aprendizaje tras recibir un tema de la Unidad Didáctica del programa de la asignatura estadística, antes de cualquier intervención (Pretest), con el fin de determinar la homogeneidad de los tres grupos de estudio. Como se observa en la Tabla 2, para el supuesto de normalidad de las calificaciones (V.D.), los respectivos Valores P del estadístico de Shapiro-Wilk para los grupos GC (grupo control), GEA (grupo experimental A) y GEB (grupo experimental B) son: 0.452, 0.226, 0.143 y los del estadístico de Kolmogorov-Smirnov son: $2.20,0.05$ y 0.08 ; como estos valores son todos mayores que el nivel de significancia $\alpha=0.01$, no se rechaza la hipótesis nula de normalidad, es decir, las calificaciones en los tres grupos se distribuyen en forma normal.

En cuanto a la homogeneidad de las varianzas, la Tabla 3 del estadístico de Levene arroja un Valor P (0.099), mayor que $\alpha=0.01$, luego las varianzas en los tres grupos son iguales. En la Figura 1 de boxplot, se puede observar la no existencia de datos atípicos, la homogeneidad en las varianzas y una pequeña aproximación a la existencia de igualdad en los promedios de las calificaciones. Cumplidos los anteriores supuestos, se hace referencia a la Tabla 4 (tabla ANOVA), y se detalla que el Valor P (0.023), es mayor al nivel de significancia $\alpha=0.01$, por lo tanto no se rechaza la hipótesis nula de igualdad de medias, es decir las calificaciones promedios en los tres grupos es igual, o dicho de otra forma, los conocimientos sobre el tema de la Unidad Didáctica del 
programa de la asignatura de estadística sin intervención alguna son los mismos, esto implica que existe homogeneidad en los conocimientos que tienen los tres grupos.

\begin{tabular}{|l|c|c|c|c|c|c|}
\hline \multicolumn{7}{|c|}{ Pruebas de normalidad } \\
\hline GRUPO & \multicolumn{2}{|c|}{ Kolmogorov-Smirnov(a) } & \multicolumn{3}{c|}{ Shapiro-Wilk } \\
\hline & Estadístico & gl & Sig. & Estadístico & gl & Sig. \\
\hline GC & 0.145 & 22 & $0.200\left(^{*}\right)$ & 0.958 & 22 & 0.452 \\
\hline GEA & 0.159 & 30 & 0.050 & 0.955 & 30 & 0.226 \\
\hline GEB & 0.156 & 28 & 0.080 & 0.944 & 28 & 0.143 \\
\hline
\end{tabular}

Tabla 2. Prueba de normalidad para la variable dependiente (calificación)

* Este es un límite inferior de la significación verdadera.

(a) Corrección de la significación de Lilliefor



Figura 1. Variable calificación por cada grupo

\section{Prueba de homogeneidad de varianzas}

En segundo lugar las pruebas estadísticas para las calificaciones pos test con la intervención de las estrategias de toma de apuntes (hoja modelo para el grupo experimental A y cuadro sinóptico para el grupo experimental B) arrojaron los siguientes resultados: 


\begin{tabular}{|c|c|c|c|}
\hline Estadístico de Levene & gl1 & gl2 & Sig. \\
\hline 2.382 & 2 & 77 & 0.099 \\
\hline
\end{tabular}

Tabla 3. Prueba de igualdad de varianzas para la variable Nota

\begin{tabular}{|l|c|r|c|c|c|}
\hline & Suma de cuadrados & \multicolumn{1}{|c|}{ gl } & Media cuadrática & F & Sig. \\
\hline Inter-grupos & 0.650 & 2 & 0.325 & 3.976 & 0.023 \\
\hline Intra-grupos & 6.290 & 77 & 0.082 & & \\
\hline Total & 6.940 & 79 & & & \\
\hline
\end{tabular}

Tabla 4. ANOVA para igualdad de medias de nota

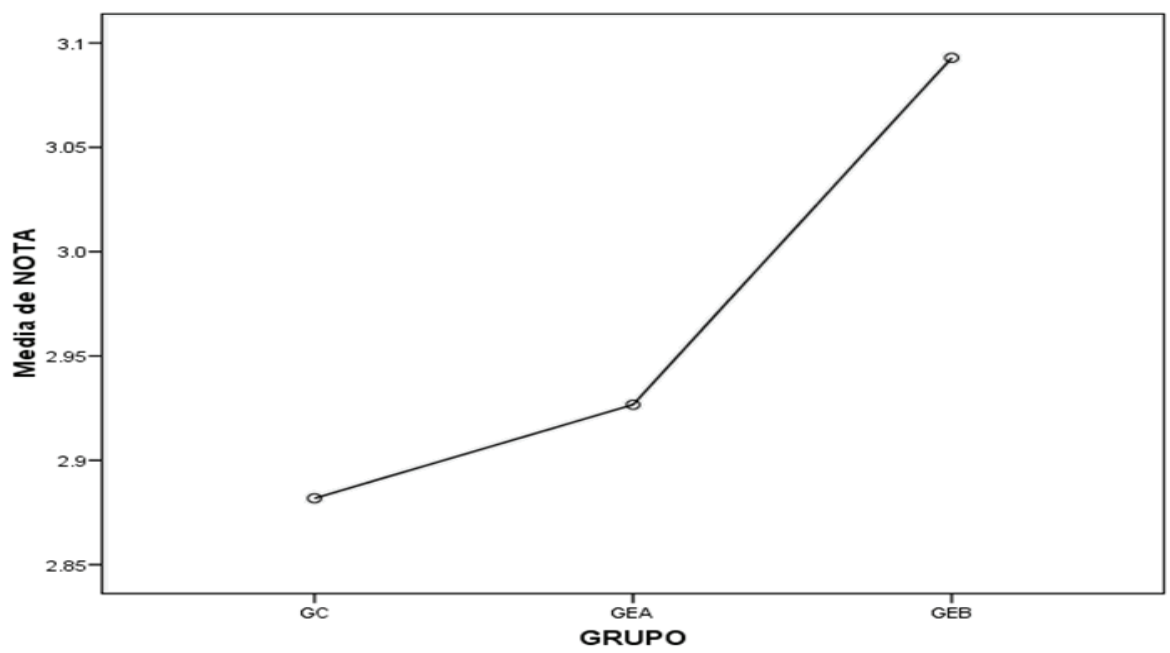

Figura 2. Medias de las notas

Los valores P de 0.196, 0.20, 0.012 para la prueba de Kolmogorov -Smirnov (Tabla V), y los valores $\mathrm{P}$ de $0.710,0.581,0.036$ respectivamente para el grupo control (GC), el grupo experimental A (GEA) y grupo experimental B (GEB) son todos mayores al nivel de significancia $\alpha=0.01$, lo que conduce a determinar la normalidad de las calificaciones en los tres grupos de trabajo. De la misma forma el valor P (0.981) de la prueba de Levene (Tabla 6), es muy superior al nivel de significancia $\alpha=0.01$, luego las varianzas para los tres grupos son homogéneas. En el diagrama Boxplot (Figura 3), 
se evidencia la disminución de la variabilidad en los tres grupos, como la no existencia de puntos atípicos. Bajo los supuestos anteriores se realizó el ANOVA de un factor para la igualdad de medias, y como lo muestra la Tabla ANOVA (Tabla 6), con un Valor P (0.000) menor al nivel de significancia $\alpha=0.01$, se rechaza la hipótesis nula y se acepta la hipótesis alterna que indica que por lo menos dos medias son diferentes.

\begin{tabular}{|l|c|c|c|c|c|c|}
\hline \multicolumn{9}{|c|}{ Pruebas de normalidad } \\
\hline \multirow{2}{*}{ GRUPO } & \multicolumn{3}{|c|}{ Kolmogorov-Smirnov(a) } & \multicolumn{4}{c|}{ Shapiro-Wilk } \\
\cline { 2 - 7 } & Estadístico & $\mathrm{gl}$ & Sig. & Estadístico & G1 & Sig. \\
\hline GC & 0.153 & 22 & 0.196 & 0.970 & 22 & 0.710 \\
\hline GEA & 0.114 & 30 & $0.200\left(^{*}\right)$ & 0.972 & 30 & 0.581 \\
\hline GEB & 0.189 & 28 & 0.012 & 0.921 & 28 & 0.036 \\
\hline
\end{tabular}

Tabla 5. Prueba de normalidad para la variable dependiente (calificación)

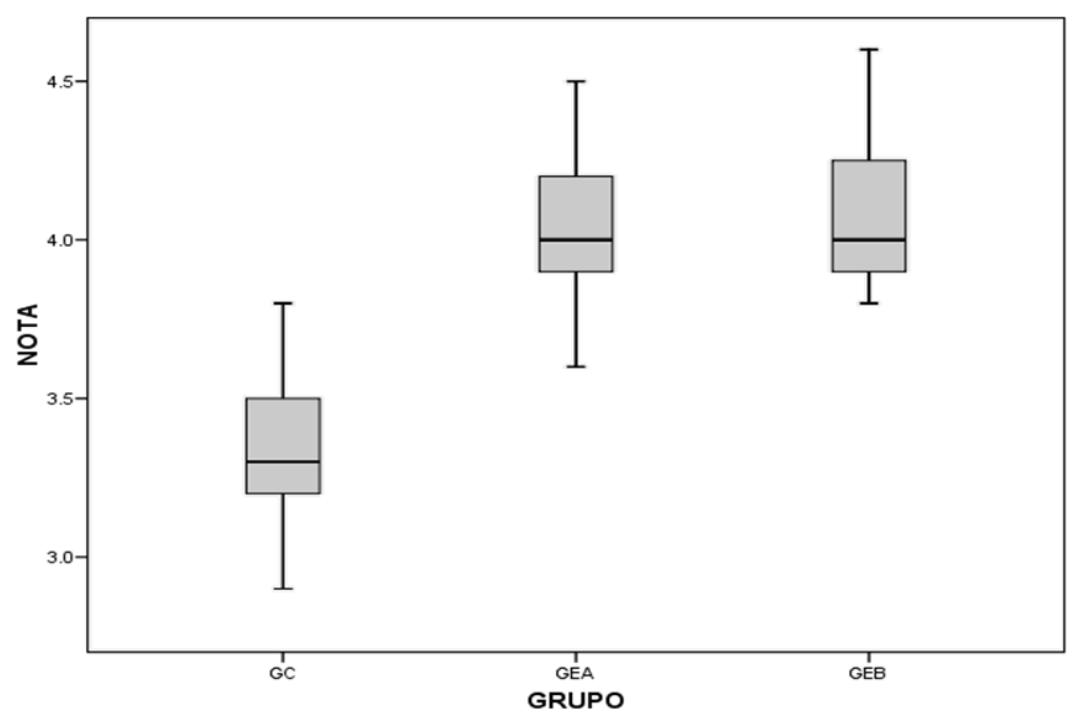

Figura 3. Variable calificación por cada grupo 


\section{Prueba de homogeneidad de varianzas}

Ahora mediante las pruebas post hoc de comparaciones múltiples (DMS y Duncan), se seleccionan cuál o cuáles pares de medias son las diferentes. La Tabla 7 arroja un Valor P (0.000) para las comparaciones del grupo control y los dos grupos experimentales, esto conduce a determinar la existencia de diferencias altamente significativas entre las medias de las calificaciones el grupo control y los dos grupos experimentales, mientras el Valor $\mathrm{P}(0.481)$ entre los dos grupos experimentales, indica igualdad entre los promedios de las calificaciones. Como el promedio del grupo control (3.336) es menor y diferente a los de los grupos experimentales, se puede concluir que las estrategias de toma de apuntes son más efectivas para el aprendizaje significativo de los estudiantes en contraste con los estudiantes que toman apuntes en forma literal.

\begin{tabular}{|c|c|c|c|}
\hline Estadístico de Levene & gl1 & gl2 & Sig. \\
\hline 0.019 & 2 & 77 & 0.981 \\
\hline
\end{tabular}

Tabla VI. Prueba de igualdad de varianzas

\begin{tabular}{|l|c|r|r|r|r|}
\hline & Suma de cuadrados & \multicolumn{1}{|c|}{ gl } & Media cuadrática & F & Sig. \\
\hline Inter-grupos & 8.547 & 2 & 4.273 & 81.600 & 0.000 \\
\hline Intra-grupos & 4.032 & 77 & 0.052 & & \\
\hline Total & 12.579 & 79 & & & \\
\hline
\end{tabular}

Tabla VII. ANOVA para igualdad de medias

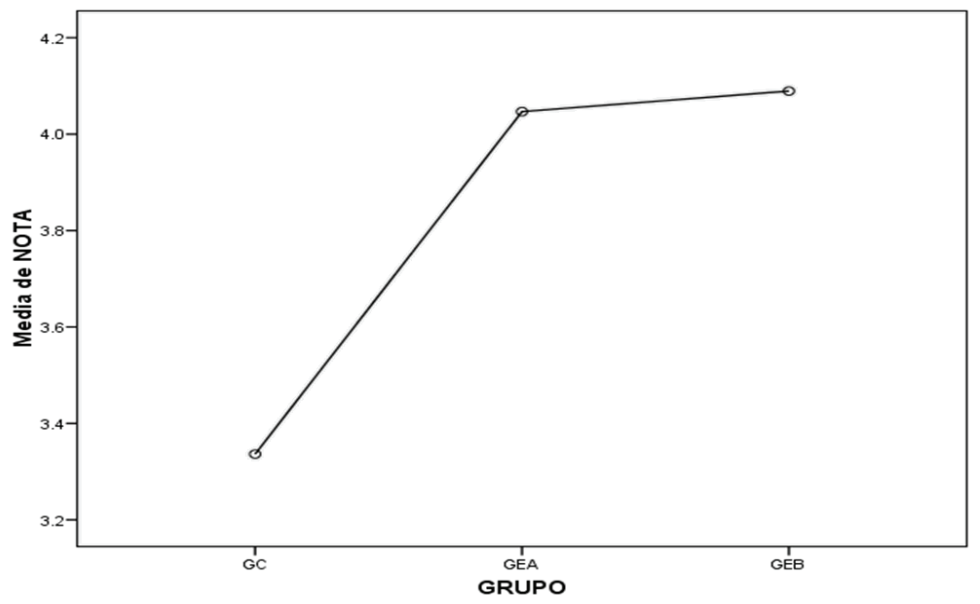

Figura 4. Gráfico de medias 
Por lo anterior, se puede concluir que en los estudiantes que utilizan estrategias para la toma de apuntes, se favorece en mayor medida el aprendizaje significativo, con respecto a los estudiantes que registran los apuntes en forma literal

\begin{tabular}{|c|c|c|c|c|c|c|c|}
\hline \multicolumn{3}{|c|}{ Variable dependiente NOTA } & \multicolumn{5}{|c|}{ Comparaciones múltiples } \\
\hline & \multirow{2}{*}{$\begin{array}{c}\text { (I) } \\
\text { GRUPO }\end{array}$} & \multirow{2}{*}{$\begin{array}{c}\text { (J) } \\
\text { GRUPO }\end{array}$} & \multirow{2}{*}{$\begin{array}{l}\text { Diferencia de } \\
\text { medidas (I-J) }\end{array}$} & \multirow{2}{*}{$\begin{array}{l}\text { Error } \\
\text { típico }\end{array}$} & \multirow{2}{*}{ Sig. } & \multicolumn{2}{|c|}{ Intervalo de confianza al $99 \%$} \\
\hline & & & & & & $\begin{array}{l}\text { Límite } \\
\text { inferior }\end{array}$ & $\begin{array}{c}\text { Límite } \\
\text { superior }\end{array}$ \\
\hline \multirow{6}{*}{ DMS } & \multirow[t]{2}{*}{$\mathrm{GC}$} & GEA & $-0.713^{*}$ & 0.642 & 0.000 & -0.880 & -0.541 \\
\hline & & GEB & $-0.529 *$ & 0.652 & 0.000 & -0.925 & -0.581 \\
\hline & \multirow[t]{2}{*}{ GEA } & GC & $0.7103 *$ & 0.642 & 0.000 & 0.541 & 0.880 \\
\hline & & GEB & -0.426 & 0.601 & 0.481 & -0.201 & 0.116 \\
\hline & \multirow[t]{2}{*}{ GEB } & $\mathrm{GC}$ & $0.7529 *$ & 0.652 & 0.000 & 0.581 & 0.925 \\
\hline & & GEA & 0.0426 & 0.601 & 0.481 & -0.116 & 0.201 \\
\hline
\end{tabular}

Tabla 8. Pruebas post hoc MMS

\begin{tabular}{|c|l|c|c|c|}
\hline \multirow{5}{*}{ Duncan } & GRUPO & $\mathbf{N}$ & \multicolumn{2}{|c|}{ Subconjunto para alfa $=\mathbf{0 . 0 1}$} \\
\hline \multirow{5}{*}{} & & $\mathbf{1}$ & $\mathbf{2}$ & $\mathbf{1}$ \\
\cline { 2 - 5 } & GC & 22 & 3.336 & \\
\cline { 2 - 5 } & GEA & 30 & & 4.047 \\
\cline { 2 - 5 } & GEB & 28 & & 4.089 \\
\cline { 2 - 5 } & Sig. & & 1.000 & 0.502 \\
\hline
\end{tabular}

Tabla 9. Pruebas post hoc DUNCAN

\section{Discusión}

Los resultados obtenidos señalan que cuando los estudiantes aplican algunas técnicas para la toma de apuntes se mejora la comprensión de los temas que cuando estos se toman solo de manera literal de la información. Sin embargo, los estudiantes no suelen recibir instrucción sobre técnicas o estrategias para tomar apuntes, por lo que deberían plantearse sesiones de orientación en este aspecto. 
El que se enseñen diversas técnicas no garantiza que luego los estudiantes las empleen cuando se enfrentan a situaciones reales de toma de datos como se observó en algunos de los sujetos del grupo experimental.

Un aspecto sobre el que se debería reflexionar, es si en el los nuevos entornos educativos en los que se está cambiando la forma de tomar de notas de clase hacia medios tecnológicos como el ordenador portátil o las tabletas digitales, siguen siendo válidas las estrategias utilizadas en la toma de apuntes tradicionales de papel y bolígrafo o se deben plantear nuevos métodos acordes con estas nuevas tecnologías que irrumpen con fuerza en la sociedad y con ella en los entornos educativos.

\section{Conclusiones}

En esta investigación, se pensó que el profesor emplea sus procedimientos didácticos con una elevada consciencia de los objetivos que se perseguían y de la función que debía desempeñar. Esta conciencia sobre el significado y el sentido de la propia actuación no se produjo, presumiblemente, entre muchos de los estudiantes participantes que se limitaron a aplicar los procedimientos aprendidos de manera «mecánica» y no estratégica buscando, por ejemplo, la reproducción literal de los apuntes del profesor. Estas diferencias entre estudiantes y profesor en cuanto al «uso estratégico» (intencional y orientado a objetivos) que hicieron de sus respectivos procedimientos podría explicar la primera conclusión a la que se llega tomando los resultados en su globalidad: para que se produzca algún grado de aprendizaje significativo en clases de tipo expositivo, lo más relevante es elegir una estrategia didáctica apropiada. Sin embargo esta última afirmación queda algo matizada cuando se comparan las ganancias que en su aprendizaje han obtenido los distintos grupos, según la misma estrategia de enseñanza que recibían y la modalidad de estrategia de anotación que empleaban. Tomando en consideración ambas variables se puede plantear que el uso de pausas expositivas por parte del docente resulta especialmente rentable cuando los estudiantes emplean las hojas-modelo y los cuadros sinópticos como estrategias de anotación.

A raíz de esta consideración se piensa que el dilema referido a la mejor manera de enseñar procedimientos de anotación no debería seguir centrándose en la dicotomía codificación vs. almacenamiento, sino que convendría reorientar la enseñanza en otra dirección, en parte apuntada al principio de estas conclusiones: asegurar que el estudiante actúa estratégicamente cuando toma notas en clase, es decir selecciona y aplica intencionalmente unos procedimientos de anotación en función de unos objetivos de aprendizaje y de las condiciones de la situación educativa que se plantea, siendo la estrategia de enseñanza que utiliza el profesor una de las condiciones más relevantes.

Una segunda implicación educativa de este estudio, íntimamente conectada con la anterior, es la constatación de que durante la toma de apuntes el estudiante puede (y debe) aprender de de forma comprensiva, y para ello es necesario que realice un esfuerzo de construcción personal apoyado en la estructura didáctica del profesor. En 
la enseñanza superior esta afirmación no es tan obvia como pudiera parecer, y las teorías pre-científicas, implícitas o explícitas, que postulan que el aprendizaje es un fiel reflejo de la enseñanza, continúan siendo mayoritarias.

En la enseñanza-aprendizaje de estrategias, como en el aprendizaje de todo tipo de contenidos educativos, el papel del docente en calidad de «mediador» es sin duda determinante. Sobre este planteamiento, ya diversos autores han insistido en otorgar al comportamiento intencional que exhibe el profesor en su clase, durante la interacción instruccional, la mayor responsabilidad en la emisión de conductas estratégicas por parte de sus alumnos (Rigney, 1978; Peterson y Swing, 1983). A la luz de los resultados obtenidos en esta investigación, se ha confirmado qué cuando el profesor asume la responsabilidad de enseñar estrategias para la toma de notas en clase se mejoran no solo los resultados en términos de nota, sino que también en el aprendizaje comprensivo de los conocimientos.

Se confía poder afirmar, a medio plazo, que el mejor «procedimiento de aprendizaje» será aquel que haga entender al alumno que no existe un «mejor procedimiento de aprendizaje», y que el camino óptimo para aprender a aprender mejor es conseguir cada vez mayores cuotas de conciencia sobre los propios procesos de aprendizaje.

\section{Referencias bibliográficas}

CASTELLÓ, M. (1993). Las estrategias de aprendizaje en el proceso de composición escrita: una propuesta de enseñanza- aprendizaje del texto argumentativo. Tesis doctoral no publicada. Universidad Autónoma de Barcelona. Barcelona: España.

COOK, T. D. Y CAMPBELL, D. T. (1979). Quasi-experimentation: design \& analysis issues for field settings. Chicago, Illinois: Rand McNally College

BADGER, R., WHITE, G., SUTHERLAND, P. Y HAGGIS, T. (2001). Note perfect: an investigation of how students view taking notes in lectures. System, 29, 405-417.

BROWN, S. Y A. GLASNER (Eds.)(2003). Evaluar en la Universidad. Problemas y nuevos enfoques. Madrid: Narcea.

ESTEVE, J. M. (2003): La tercera revolución educativa. La educación en la sociedad del conocimiento. Barcelona: Paidós.

ENRÍQUEZ, A. Y RENTERÍA, E. (2007). Estrategias de aprendizaje para la empleabilidad en el mercado del trabajo de profesionales recién egresados. Universitas Psychologica, 6(1), 89-103.

FERNÁNDEZ, A. (2006). Metodologías activas para la formación de competencias. Educatio Siglo XXI, 24, 35-56.

GIBSS, G. (2003). Uso estratégico de la evaluación en el aprendizaje. En Brown, S. y. Glasner, A. (Eds.), Evaluar en la Universidad. Problemas y nuevos enfoques (pp. 61-76). Madrid: Narcea. 
HARTLEY, J. Y DAVIES, I. K. (1978). Note-Taking: A Critical Review. Programmed Learning and Educational Technology, 15, 207-224.

IBARRA, M. S. Y RODRÍGUEZ, G. (2010). Aproximación al discurso dominante sobre la evaluación del aprendizaje en la universidad. Revista de Educación, 351, 385-407.

KIEWRA, K. A. (1989). A review of note-taking: the encoding-storage paradigm and beyond. Educational Psychology Review, 1(2), 147-172.

KIRKGÖZ, Y. (2010). Promoting students' note-taking skills through task-based learning. Procedia. Social and Behavioral Sciences, 2, 4346-4351.

KOBAYASHI, K. (2005). What limits the encoding effect of note-taking? A metaanalytic examination. Contemporary Educational Psychology, 30, 242-262.

MUÑOZ, E. Y GÓMEZ, J. (2005). Enfoques de aprendizaje y rendimiento académico de los estudiantes universitarios. Revista de Investigación Educativa, 23(2), 417432.

ROSÁRIO, P., MOURÃO, J., NÚÑEZ, J., GONZÁLEZ-PIENDA, J., SOLANO, P. Y VALLE, A. (2007). Eficacia de un programa instruccional para la mejora de procesos y estrategias de aprendizaje en la enseñanza superior. Psicothema, 19 (3), 422-427.

PÉREZ, M. V., VALENZUELA, M. F., DÍAZ, A., GONZÁLEZ-PIENDA, J. A. Y NÚÑEZ, J. C. (2011). Disposición y enfoques de aprendizaje en estudiantes universitarios de primer año. Universitas Psychologica, 10 (2), 441-449.

PETERSON, P. Y SWING, S. (1983). Problems in classroom implementation of Cognitive Strategy Instruction. En Pressley, M. y Levin, J. (Eds.), Cognitive Strategy Research. Educational Applications. Nueva York: Springer-Verlag.

RICKARDS, J. P. (1979). Note-Taking: Theory and Research. Improving Human Performance Quarterly, 8(3), 152-161.

RIGNEY, J. (1978). Learning strategies: A theoretical perspective. En O’Neil, H. (Ed.): Learning Strategies. Nueva York: Academic Press.

SLOTTE, V. Y LONKA, K. (1999). Review and process effects of spontaneous notetaking on text comprehension. Contemporary Educational Psychology, 24, 1-20.

TENG, H. C. (2011). Exploring note-taking strategies of EFL listenerst. Procedia. Social and Behavioral Sciences, 15, 480-484.

VAN METER, P., YOKOI, L. Y PRESSLEY, M., (1994). College students' theory of note-taking derived from their perceptions of note-taking. Journal of Educational Psychology, 86, 323-338.

UNESCO. (2001). Séptima reunión del Comité Regional Intergubernamental del Proyecto Principal de Educación en América Latina y el Caribe: Informe final. Santiago: Unesco-Chile. 
WARD, N. Y TATSUKAWA, H. (2003). A tool for taking class notes. Int. J. HumanComputer Studies, 59, 959-981.

\title{
Correspondencia con los autores:
}

Dagoberto SALGADO-HORTA

Facultad Ciencias de la Educación, Universidad del Tolima

Calle 42 Barrio Santa Helena Parte Alta

e-mail: dsalgadoh@ut.edu.co

Teléfono: 2771212 ext. 9318 - 2785374

\author{
Alexander MAZ-MACHADO \\ Departamento de Matemáticas, Universidad de Córdoba \\ C/San Alberto Magno s/n 1400 \\ e-mail: ma1mamaa@uco.es \\ Teléfono: 679596449
}

